\title{
Correction to: CLE2 regulates light-dependent carbohydrate metabolism in Arabidopsis shoots
}

\author{
Dichao Ma ${ }^{1} \odot$. Satoshi Endo ${ }^{1} \cdot$ Shigeyuki Betsuyaku ${ }^{2,3} \cdot$ Akie Shimotohno $^{1} \cdot$ Hiroo Fukuda $^{1}$
}

Published online: 2 April 2021

(c) Springer Nature B.V. 2021

\section{Correction to: Plant Molecular Biology (2020) 104:561-574 https://doi.org/10.1007/s11103-020-01059-y}

In the above mentioned publication, the online Supplementary file1 (PDF $36 \mathrm{~kb}$ ) was incomplete and contained the first page only. The original article has been corrected.

Publisher's Note Springer Nature remains neutral with regard to jurisdictional claims in published maps and institutional affiliations.

The original article can be found online at https://doi.org/10.1007/ s11103-020-01059-y.

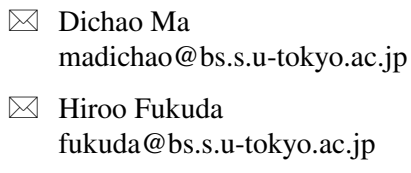

1 Department of Biological Sciences, Graduate School of Science, The University of Tokyo, 7-3-1 Hongo, Bunkyo-ku, Tokyo 113-0033, Japan

2 Faculty of Life and Environmental Sciences, University of Tsukuba, 1-1-1 Tennodai, Tsukuba, Ibaraki 305-8577, Japan

3 Microbiology Research Center for Sustainability, University of Tsukuba, 1-1-1 Tennodai, Tsukuba, Ibaraki 305-8572, Japan 